First publ. in: Nature 404 (2000), pp. 29-31

Cognitive neuroscience

\title{
Imaging in the fourth dimension
}

Thomas Elbert and Andreas Keil

$\mathrm{T}$ he 1990s, the decade of the brain, saw enormous developments in neuroimaging. Structural details of the brain can now be reconstructed non-invasively as three-dimensional images; and small, taskrelated changes in cerebral blood flow, even in the deepest recesses of the brain, can be seen. The principles of the functional organization of the brain are being uncovered, and it seems that not only initial processing stages but also complex aspects of perception and cognition can be mapped onto brain structures $^{1,2}$. Nevertheless, cognitive neuroscientists find themselves in an odd, but perhaps not surprising, situation. As the number of studies increases, so does the number of conflicting results. On page 80 of this issue, Patel and Balaban ${ }^{3}$ provide an example of what has been missing in neuroimaging - a new approach that adds time as the fourth dimension.

Usually, three-dimensional images of cerebral blood flow, metabolic changes or the activity of populations of neurons are

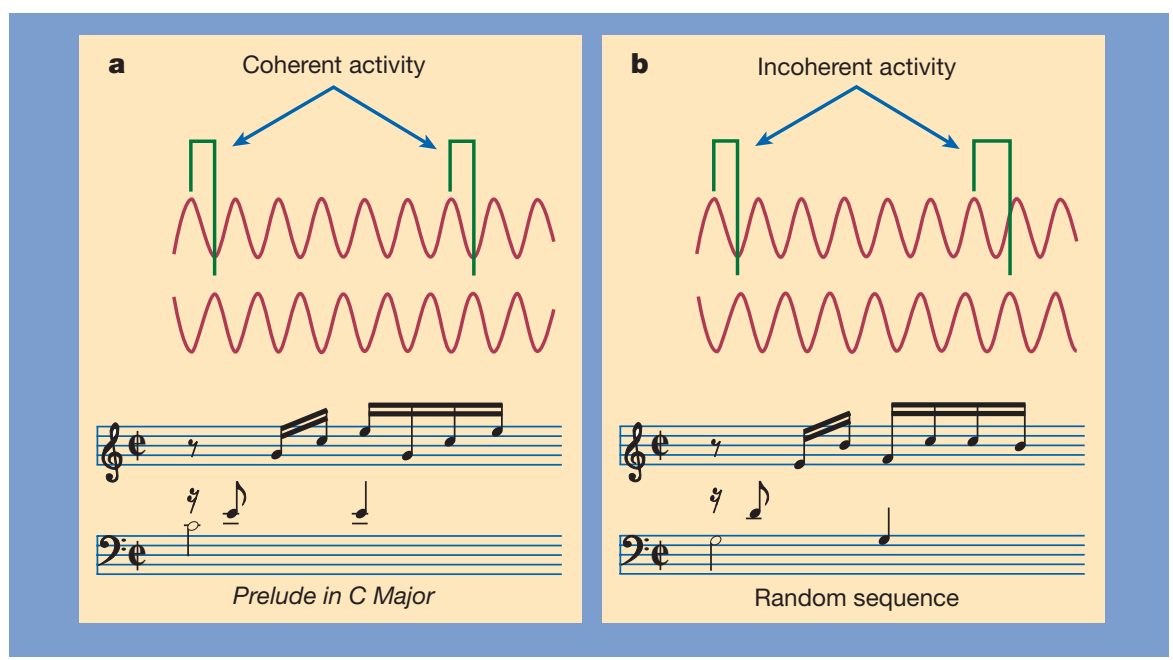

Figure 1 Music and random notes produce different neural responses. Oscillatory brain activity evoked by repetitive auditory stimulation shows different temporal relationships between widely separated recording sites, depending on stimulus properties. a, Patel and Balaban ${ }^{3}$ found that predictable, melody-like tone sequences are associated with coherent activity and more constant phase lags of oscillatory responses recorded from distant channels. This synchronization of brain activity in distant areas (the traces shown above the music) may reflect perceptual integration. The example shown here, Bach's Prelude in C Major, should produce high coherence. b, In contrast, a random sequence of tones showing identical rhythmic structure but no melody should produce incoherent activity of distant channels, with less synchronization between brain regions. 
acquired during a given task or perceptual activity (while the subject is listening to Bach's Prelude in C Major, for example). The temporally static image can account only for a static cerebral response. But the brain's response is actually dynamic and selforganizing over time. So the perception of music and its neural code must be reflected in the neural dynamics - in both space and time. It is not only important to identify the neurons, neuronal assemblies or brain regions that respond to a given input. We must also develop techniques that allow the systematic classification of the temporal dynamics underlying elements of information processing. But adding time as the fourth dimension to three-dimensional space is not easy.

Patel and Balaban ${ }^{3}$ study brain dynamics using stimulus-related magnetoencephalographic responses. Subjects hear different sequences of tones that are switched on and off in rapid, 40-Hz sequences. Examination of the stimulus-related brain response that cycles at $40 \mathrm{~Hz}$, the so-called steady-state response, allowed Patel and Balaban to determine how the timing of neural responses varied with different tone sequences. They found a relationship between the phase (but not the signal power) of the steady-state response and the frequency of the acoustic stimulus. That is, the timing of the neuronal response depended on the properties of the stimulus.

Intriguingly, these phase fluctuations vary with the structures of the tone sequences. Between-site phase coherence, which indicates synchronized activity between brain areas, was most pronounced for tone sequences that resembled melodies. Generalizing this outcome, the Prelude in $C$ Major should produce higher inter-channel phase coherence than the same tones shuffled in random sequence (Fig. 1). Would Bach do better than the Beatles? We do not know, but now we can study how limited brain regions track the changes in pitch of auditory sequences as a piece of music is played.

Steady-state responses are a valuable tool for monitoring activity in different sensory modalities. To exploit this approach fully, we will need to understand how such brain responses are produced. In general, if the interval between successive stimuli is short enough, the transient evoked response to one stimulus will not have died away before the next stimulus is delivered. The compound response that appears is the steadystate response. There are various ways in which transient responses can sum over time to produce a steady-state response, and these fall into two groups. For a linear system, transient and steady-state descriptions of the system's behaviour are equivalent, and a simple superposition of transient evoked responses with the appropriate time lags should fully predict the steady-state respons$e^{4}$. However, neural assemblies are nonlinear elements. If a nonlinear system is stimulated periodically, harmonics, combination frequencies and subharmonic components may evolve 5 .

Neither of these simple principles of organization accounts for the observation by Balaban and Patel that the phase of the steady-state response follows the pitch of the auditory stimulus more strongly for scales than for melodies. Obviously, higher areas of brain influence the auditory cortex and related structures by 'top-down' processes, tuning their responses according to contextual cues and previous learning. So coupled oscillations between higher-order and sensory cortices may explain why what sounds like noise to an adult is music to the ears of a teenager.

Attempts to segregate brain function into distinct modules are limited because the nervous system tends to operate through the intercommunication of task-relevant subsystems. So the simplistic modular approach needs to be complemented by modelling, in space and time, the network that incorporates the different modules. In the visual system, several different types of discrimination can be processed in the same small area of cortex. A similar phenomenon has been seen in the motor system ${ }^{6}$. Likewise, a single type of stimulation of two digits can produce two opposite, use-dependent effects on the spatial relationship of the cortical representations of the digits, depending on the nature of the discrimination condition used $^{7}$. In other words, multiple maps, specific to different modes of discrimination or tasks, share the same region of cortex. So the three-dimensional modular approach provides us with seemingly conflicting results: there are many three-dimensional shadows in a four-dimensional world. It is time to add time!

Thomas Elbert and Andreas Keil are in the Department of Psychology, University of Konstanz, D-78457 Konstanz, Germany.

e-mails: thomas.elbert@uni-konstanz.de andreas.keil@uni-konstanz.de

1. Frith, C. D. \& Friston, K. J. in Cognitive Neuroscience (ed. Rugg, M. D.) 169-195 (MIT Press, Cambridge, Massachusetts, 1997).

2. Elbert, T. in Magnetism in Medicine (eds Andrä, W. \& Nowak, H.) 190-262 (Wiley, New York, 1998).

3. Patel, A. \& Balaban, E. Nature 404, 80-84 (2000).

4. Gutschalk, A. et al. Clin. Neurophysiol. 110, 856-868 (1999).

5. Pantev, C., Roberts, L. E., Elbert, T., Ross, B. \& Wienbruch, C Hearing Res. 101, 62-74 (1996)

6. Karni, A. et al. Nature 377, 155-158 (1995).

7. Braun, C., Schweizer, R., Elbert, T., Birbaumer, N. \& Taub, E. J. Neurosci. 20, 446-450 (2000). 